\title{
Lattice dynamics of noble and transition metals
}

\author{
Barry L Fielek ${ }^{\dagger}$ \\ University of Michigan, Ann Arbor, Michigan, USA
}

Received 26 June 1974, in final form 19 August 1974

\begin{abstract}
A simple model for the calculation of the phonon dispersion curves of the noble and transition metals is proposed. The model uses the concept of a rigid shell moving relative to its nucleus to simulate the response of the d electrons to the nuclear motion, short range central forces for the interaction between the ion cores, and a screened pseudopotential for the conduction electron response. The most general form of the model considered for fcc metals contains six parameters, three of which may be obtained from the adiabatic elastic constants and the remainder from a least square fit to the experimental frequencies. Preliminary applications of the model containing five parameters made for copper and nickel have resulted in fits to the experimental dispersion curves which are within 2 and $2.5 \%$. respectively.
\end{abstract}

\section{Introduction}

From first principles, within the harmonic approximation, the vibrational energy of a system of atoms in a solid can be written as (Joshi and Rajagopal 1968)

$$
V=V_{\mathrm{ii}}-V_{\mathrm{ie}}(\epsilon-1) / \epsilon
$$

or

$$
V=V_{\mathrm{ii}}-V_{\mathrm{ie}}+V_{\mathrm{ie} / \epsilon}
$$

where the first term is the second order change in the ion-ion potential energy with respect to the displacement of the ions, the next term is the second order change in the bare electron-ion potential energy, and the third term (which is the second order change in the bare electron-ion potential energy divided by the dielectric function $\epsilon$ ) is the screened electron-ion potential energy arising from the interaction between the electrons. For simple metals, the atom is divided into nearly free conduction electrons and a well localized ion composed of the nucleus plus the remaining inner core of electrons. Under these assumptions, the ion-ion term is produced by Coulomb interactions between point charges, while the bare electron-ion term results from interactions between a conduction electron and the pseudopotential of an ion.

The direct application of equation (1) to the calculation of the phonon dispersion curves of simple metals is difficult. The major reasons for this are a lack of knowledge as to how to treat the interactions between electrons which determine the dielectric function, and how to describe the ionic potential that an electron sees within the solid. The latter has been handled relatively successfully for simple metals by model potentials

† Present address: 6610 Sutton Rd, Whitmore Lake. Michigan 48189, USA. 
based on first principles pseudopotential theory (Heine et al 1970). In these calculations the ionic potential is parametrized. The parameters are obtained from experimental atomic spectrum energies or from other properties such as the elastic constants or the experimental phonon frequencies themselves. Because of the difficulties encountered in first principles calculations, considerable effort has been devoted to the development of phenomenological theories in which reasonable guesses are made as to the nature of the forces acting between nuclei (de Launay 1956).

From equation (2), it is seen that the bare electron-ion interaction reduces the effect of the long range Coulomb interaction between the ions. Also, from first principles calculations, it is found that the electron-ion interaction reduces the ion-ion interaction to short range forces between ions (Harrison 1966). Furthermore, from Born-von Karman force constant models, it is found that only a few force constants between a few nearest neighbours are required to obtain a good overall fit to the experimental phonon dispersion curves of the simple metals, the noble metals and some transition metals. However, for a detailed fit, several more force constants (extending out to many neighbours in the case of some transition metals) are required. This indicates that, as well as the short range forces which reproduce the overall structure of the dispersion curves, there are long range forces of lesser magnitude present which produce the finer structure of the dispersion curves (Woods 1972). These long range forces are believed to result from the screened electron-ion potential energy term in equation (2) which reduces the effect of the bare electron-ion term on the ion-ion interaction term.

Several phenomenological models have been proposed which attempt to reduce the number of force constants required to fit a phonon dispersion curve by phenomenologically incorporating the long range effects of the conduction electrons. The best of these models is the one proposed by Krebs (1965). This model contains central forces between only a few nearest neighbours. For simple metals, the number of force constants in most cases is sufficiently small to facilitate their evaluation from the elastic constants. The model also contains the following expression to take account of the long range effects of the conduction electrons:

$$
A G(\boldsymbol{q})=A \sum_{H}\left[\frac{(\boldsymbol{q}+\boldsymbol{H})(\boldsymbol{q}+\boldsymbol{H}) \cdot e g^{2}\left(|\boldsymbol{q}+\boldsymbol{H}| \boldsymbol{R}_{0}\right)}{|\boldsymbol{q}+\boldsymbol{H}|^{2}+\hat{\lambda} \epsilon(q+H) / \lambda_{\mathrm{TF}}}-\frac{\boldsymbol{H} \boldsymbol{H} \cdot e g^{2}\left(H R_{0}\right)}{\boldsymbol{H}^{2}+\lambda \epsilon(H) / \hat{\lambda}_{\mathrm{TF}}}\right]
$$

where $\boldsymbol{q}$ is the wave vector, $\boldsymbol{H}$ is a reciprocal lattice vector, $\boldsymbol{e}$ is the polarization vector, $\epsilon$ is the dielectric function (in the case of the Krebs model, the Lindhard dielectric function), $\lambda_{\mathrm{TF}}$ is the Thomas-Fermi screening parameter, $g(x)=(\sin x-x \cos x) / 3 x^{3}, A$ is an unknown parameter which can be obtained from the elastic constants, $i$ is the BohmPines screening parameter and $R_{0}$ is the Wigner-Seitz radius obtained from the unit cell volume. The expression in equation (3) with $A$ equal to $4 \pi e^{2} /(\mathrm{vol}$ unit cell) and $\lambda$ equal to $\lambda_{\mathrm{TF}}$ is the Fourier transform of a screened electron-ion pseudopotential energy obtained by assuming a pseudopotential of the form (Joshi and Rajagopal 1968)

$$
V(r)= \begin{cases}A e / r & r>R_{0} \\ A\left(3 e / 2 R_{0}-e r^{2} / 2 R_{0}^{3}\right) & r<R_{0}\end{cases}
$$

This is the potential produced by a uniform spherical charge distribution of radius $R_{0}$. As in first principles calculations, the most questionable terms are the bare electronion pseudopotential and the Lindhard dielectric function. Krebs tried to improve the Lindhard dielectric function by using the Bohm-Pines screening parameter in place of 
the Thomas-Fermi screening parameter. The Krebs model reproduces the phonon dispersion curves of the simple metals (eg Na and K (Krebs 1965)) quite well. On comparing the Krebs model with equation (2), it can be inferred that for simple metals the first two terms of equation (2) are well reproduced by central forces between a few nearest neighbours, and the third term is reproduced by the expression in equation (3).

The Krebs model does not give a good fit to the phonon dispersion curves of non simple metals, such as the noble (Shukla 1964) or transition metals (Hautecler and van Dingenen 1965). The reason for this is believed to be that the outermost $\mathrm{d}$ electrons are not rigidly bound to, nor nearly free from, their respective nucleus but are loosely bound to it. Hence, the outermost d electrons cannot be treated as rigid inner core electrons nor as nearly free conduction electrons.

In recent years, several authors (Sinha 1969, Hanke 1973) have proposed treating the outermost $d$ electrons as tightly bonded electrons. In these treatments, the $d$ electrons are allowed to respond to the displacement of the ion cores. The secular equations obtained for the phonon frequencies can be shown to be similar in form to those obtained from the shell model originally proposed by Dick and Overhauser (1958). The earlier results of Sinha's work led Hanke and Bilz (1971) to apply the breathing shell model of Schroder (1966) to some noble and transition metals. Their model contained seven parameters which were fitted to the elastic constants and four experimental frequencies. The resulting fits for the noble and fcc transition metals considered were quite good. More recently, Hanke (1973) has proposed a first principles approach similar to Sinha (1969) in which again the resulting secular equations are found to have the form of those obtained through the shell model. He has applied his model to the fcc transition metals Pd and Ni (Hanke 1973) and obtained a fairly good representation of the overall structure of the dispersion curves. In order to reduce the computational work, several approximations with regard to the $d$ band structure and ion core potential seen by the d electrons were made.

Based on the above results, this paper proposes a very simple model similar to the Krebs model, and using the concept of a shell for the response of the $\mathrm{d}$ electrons to the lattice motion. In the next section, the theory for the proposed model is given. The model itself is described in $\$ 3$, and $\$ 4$ discusses the application of the model to copper and nickel.

\section{Theory}

For a non simple metal, the atoms in the solid are assumed to be composed of three entities: the conduction electrons, which are spread throughout the solid, a shell of outermost $\mathrm{d}$ electrons and the ion core (composed of the nucleus plus the remaining core electrons which are taken to move rigidly with the nucleus). Under this division, equation (1) can be written as

$$
V=V_{\mathrm{cc}}+V_{\mathrm{dd}}-(\epsilon-1)\left(V_{\mathrm{ce}}+V_{\mathrm{de}}\right) / \epsilon+V_{\mathrm{cd}}
$$

or

$$
V=V_{\mathrm{cc}}+V_{\mathrm{dd}}-V_{\mathrm{ce}}-V_{\mathrm{de}}+V_{\mathrm{ce}} / \epsilon+V_{\mathrm{de}} / \epsilon+V_{\mathrm{cd}}
$$

where $V_{\mathrm{cc}}$ is the second order change in the potential energy with respect to the displacement of the ion cores and is due to the interactions between the ion cores. $V_{\mathrm{dd}}$ is the second order change in the potential energy between the d electrons on neighbouring 
ions due to a relocation of the d electron charge distributions. $V_{\mathrm{ce}}$ and $V_{\mathrm{de}}$ are the second order changes arising from the interaction between the conduction electrons and the ion core and the d electrons, respectively, due to relocation of the ion core and d electrons, respectively. $\epsilon$ is the dielectric function arising from the interaction between the conduction electrons. $V_{\text {cd }}$ is the change in potential energy arising from the interaction between the ion core and its $\mathrm{d}$ electrons as a result of displacement of the ion core or redistribution of the d electron charge distribution. Since the ion cores are well localized the interactions between ion cores can be taken as Coulomb interactions between point charges. Due to the distribution of the conduction electrons throughout the metal and the finite distribution of the core and d electrons, the interaction between the conduction electrons and the ion core or the d electrons can be considered as an interaction of the conduction electrons with the respective pseudopotentials arising from the ion core and $\mathrm{d}$ electrons. The interaction between neighbouring $\mathrm{d}$ electrons would contain two terms: a Coulomb interaction between the neighbouring d electron charge distributions and an exchange interaction between nearest neighbour $d$ electrons produced by the overlapping of the nearest neighbour $d$ electrons. The exchange interaction tends to localize the $\mathrm{d}$ electrons by reducing the overlapping between neighbouring $\mathrm{d}$ electrons. The separation caused by the exchange interaction reduces the Coulomb interaction between the neighbouring $\mathrm{d}$ electrons by increasing their interaction distance. Thus, the exchange potential energy will reduce the Coulomb potential energy between neighbouring d electrons.

The potential energy terms in equation (5) are dependent on the product of the displacements of the ion cores and the d electron charge distributions. Noting these dependences and reordering equation (5), the following equation is obtained

$$
\begin{array}{r}
V=V_{\mathrm{cc}}\left(U_{\mathrm{c}} U_{\mathrm{c}^{\prime}}\right)-V_{\mathrm{ce}}\left(U_{\mathrm{c}} U_{\mathrm{c}^{\prime}}\right)+V_{\mathrm{ce}}\left(U_{\mathrm{c}} U_{\mathrm{c}^{\prime}}\right) / \epsilon+V_{\mathrm{dd}}\left(U_{\mathrm{s}} U_{\mathrm{s}^{\prime}}\right) \\
-V_{\mathrm{de}}\left(U_{\mathrm{s}} U_{\mathrm{s}^{\prime}}\right)+V_{\mathrm{de}}\left(U_{\mathrm{s}} U_{\mathrm{s}^{\prime}}\right) / \epsilon+V_{\mathrm{cd}}\left(U_{\mathrm{c}} U_{\mathrm{s}}\right) .
\end{array}
$$

The first three terms are analogous to the three terms in equation (2). Hence, these interactions should be analogous to those for simple metals. Since the Krebs model reproduces the interactions for simple metals well, it should be correct to replace the first three terms in equation (7) with the Krebs model. The next two terms are similar to the first two for the ion cores except for the addition of the exchange effect in the $V_{\mathrm{dd}}$ term which further reduces the Coulomb interactions along with the term $V_{\mathrm{de}}$ for the interaction between the conduction electrons and the d electrons. Thus, the two terms, $V_{\mathrm{dd}}$ and $V_{\mathrm{de}}$, should give rise to shorter range forces than the first two terms in equation (7). Hence, it should be sufficient to replace these two terms by a central force between nearest neighbour $d$ electrons only. The screened interaction term between the $d$ electrons and conduction electrons should be similar to the screened interaction term between the ion cores and the conduction electrons, since both are due to screened pseudopotentials which arise from the inner core electrons of the ion. Thus, the second three terms are assumed to be analogous to the first three and are replaced with the Krebs model depending on the $\mathrm{d}$ electron charge distributions relative to the ion core locations and containing a central force between nearest neighbour $d$ electron distributions only. The last term is the potential energy produced by the interaction between the d electrons and their respective ion core, and depends on the position of the $\mathrm{d}$ electrons and their ion core. If this interaction is analogous to the interaction in insulators, which leads to electronic polarization, then the results of the shell model calculations 
on insulators indicate that the effects of this term can be reproduced by an isotropic force between the ion core and a rigid shell representing the d electrons.

\section{Model}

The following substitutions were now made in equation (7):

(1) The first two terms are replaced by central forces between nearest and next nearest ion cores represented by the force constants, $x_{1}, x_{2}$.

(2) The fourth and fifth terms are replaced by a central force between nearest neighbour rigid shells representing the d electrons around an ion core, and with a force constant denoted by $S$.

(3) The third term is replaced by the expression given in equation (3) multiplied by the product of the ion core displacements.

(4) The sixth term is replaced by the expression in equation (3) with $A$ replaced by $A^{\prime}$ and multiplied by the product of the shell displacements.

The negative of the force on an ion core can be obtained by taking the gradient of the potential energy given by equation (7) with respect to the displacement of the ion core. The negative of the force on a shell can be obtained by taking the gradient with respect to the shell displacement.

Considering the case of a fcc crystal structure with longitudinal motion along the symmetric direction (100), the following equations can be obtained for the natural frequencies of an ion core and its shell: .

$$
\begin{aligned}
& M \omega^{2} U_{\mathrm{c}}=-\left[8 \alpha_{1} \sin ^{2}(q a / 2)+4 x_{2} \sin ^{2}(q a)+A G(q)+K\right] U_{\mathrm{c}}+K U_{\mathrm{s}} \\
& m \omega^{2} U_{\mathrm{s}}=-\left[8 \operatorname{Sin}^{2}(q a / 2)-A^{\prime} G(q)+K\right] U_{\mathrm{s}}+K U_{\mathrm{c}}
\end{aligned}
$$

where $M$ and $m$ are the masses of the ion core and shell, respectively, $U_{\mathrm{c}}$ and $U_{\mathrm{s}}$ are the maximum displacements of the ion core and shell, respectively, $2 a$ is the lattice constant, $\omega$ is the natural angular frequency, and $q$ is the wavevector. On applying the adiabatic approximation to the shell, equations (8) and (9) can be reduced to equation (10) for the ion core motion. Applying the same reduction of the equations for the other symmetric directions, the following equations are obtained:

\section{(a) Direction (100)}

Longitudinal branch

$$
\begin{gathered}
M \omega^{2}=-\left[8 \alpha_{1} \sin ^{2}(q a / 2)+4 \alpha_{2} \sin ^{2}(q a)+K-A G(q)\right] \\
+K^{2} /\left[K+8 S \sin ^{2}(q a / 2)-A^{\prime} G(q)\right]
\end{gathered}
$$

Transverse branches $(010)$ or $(001)$

$$
M \omega^{2}=-\left[4 \alpha_{1} \sin ^{2}(q a / 2)+K-A G(q)\right]+K^{2} /\left[K+4 S \sin ^{2}(q a / 2)-A^{\prime} G(q)\right]
$$


(b) Direction (110)

Longitudinal branch

$$
\begin{gathered}
M \omega^{2}=-4 x_{1}\left[\sin ^{2}(q a / 2 \sqrt{2})+\sin ^{2}(q a / \sqrt{ } 2)\right]-4 x_{2} \sin ^{2}(q a / \sqrt{ } 2)-K+A G(q) \\
+K^{2} /\left\{K+4 S\left[\sin ^{2}(q a / 2 \sqrt{2})+\sin ^{2}(q a / \sqrt{ } 2)\right]-A^{\prime} G(q)\right\}
\end{gathered}
$$

Transverse branch $\mathrm{T}_{2}(001)$

$$
\begin{aligned}
& M \omega^{2}=-8 x_{1} \sin ^{2}(q a / 2 \sqrt{2})-K+A G(q) \\
& +K^{2} /\left[K+8 S \sin ^{2}(q a / 2 \sqrt{2})-A^{\prime} G(q)\right]
\end{aligned}
$$

Transverse branch $T_{1}(1-10)$

$$
\begin{gathered}
M \omega^{2}=-4 \alpha_{1} \sin ^{2}(q a / 2 \sqrt{2})-4 x_{2} \sin ^{2}(q a / \sqrt{2})-K+A G(q) \\
+K^{2} /\left[K+4 S \sin ^{2}(q a / 2 \sqrt{2})-A^{\prime} G(q)\right]
\end{gathered}
$$

(c) Direction (111)

Longitudinal branch

$$
\begin{gathered}
M \omega^{2}=-8 x_{1} \sin ^{2}(q a / \sqrt{3})-4 x_{2} \sin ^{2}(q a / \sqrt{3})-K+A G(q) \\
+K^{2} /\left[K+8 S \sin ^{2}(q a / \sqrt{ } 3)-A^{\prime} G(q)\right]
\end{gathered}
$$

Transverse branches $(1-10)$ or $(11-2)$

$$
\begin{gathered}
M \omega^{2}=-2 \alpha_{1} \sin ^{2}(q a / \sqrt{3})-4 x_{2} \sin ^{2}(q a / 3)-K+A G(q) \\
+K^{2} /\left[K+2 S \sin ^{2}(q a / \sqrt{3})-A^{\prime} G(q)\right] .
\end{gathered}
$$

These equations contain six unknown parameters. Three of these parameters can be related to the three elastic constants by comparing the long wavelength limit of the above equations with the Christoffell equations for a continuum. In this limit the waves are sound waves. The propagation of these waves is thermodynamically an adiabatic process (Zemansky 1951). Thus the elastic constants to be used in the Christoffell equations are the adiabatic ones. The following results are obtained

$$
\begin{aligned}
& \alpha_{1}+S=-2 C_{44} a \\
& \alpha_{2}=-\frac{1}{2}\left(C_{11}-C_{12}-C_{44}\right) a \\
& A+A^{\prime}=\left(C_{12}-C_{44}\right) 2 a^{3} \lambda
\end{aligned}
$$

where $C_{11}, C_{12}$ and $C_{44}$ are the adiabatic elastic constants. It is noted that the force constant $K$ does not appear in these expressions, and the force constant $S$ contributes to the elastic constants. The reason for this is that the shell moves rigidly with the ion core at long wavelengths. It may also be noted that assuming central forces between nearest neighbour ion cores only, imposes a restriction on the elastic constants given by putting the left hand side of equation (18) equal to zero. This relationship is not found to be satisfied by the elastic constants of the noble or transition metals. 
$A^{\prime}$ can be written as a multiple $P$ of the right hand side of equation (19). $A$ and $A^{\prime}$ are then expressed as follows:

$$
\begin{aligned}
& A^{\prime}=P\left(C_{12}-C_{44}\right) 2 a^{3} \lambda \\
& A=(1-P)\left(C_{12}-C_{44}\right) 2 a^{3} \lambda
\end{aligned}
$$

where $0 \leqslant P \leqslant 1$. With respect to the previous comparison made between terms in equations (10) and (7), the value of $P$ equal to zero implies that there is no interaction between the conduction electrons and the shell and hence no screening of the interaction between the shells. On the other hand, it may imply that the dielectric function arising in response to the interaction between the shell and conduction electrons is very large and different from the dielectric function for the interaction between the conduction electrons and the ion cores. Or, the value of $P$ equal to zero could imply that the screened potential energy between the conduction electrons and the shell electrons when added to the shell-shell plus bare shell-conduction electron potential energies gives the central force between nearest neighbour shells. This would mean that the interaction between the shell electrons and the conduction electrons is not similar to the interaction between the ion core and the conduction electrons as assumed in equations (7)-(15). The first two conditions are unrealistic.

If an attempt is made to fit the three parameters to the experimental frequencies on the boundary of the Brillouin zone for the $(100)_{L},(100)_{T}$ and $(111)_{L}$ modes, it is found that the only value of $K$ and $S$ which satisfy the three equations for these points, when $P$ is equal to zero, is zero for both $K$ and $S$ whatever the values of the frequencies that are used in equations (10), (11) and (15). This case thus reduces equations (10)-(15) to those for the Krebs model, which is known to give a poor fit to the experimental frequencies.

For the case $P$ equal to one, the three above implications could be made for the interaction between the conduction electrons and the ion cores. The first two are unrealistic. The third will be consistent for simple and non simple metals if the screened conduction electron-ion potential energy for simple metals, as well as for non simple metals, is due to the outermost electrons of the ion, and if the interaction between the conduction electrons and the inner core electrons just contributes to the formation of short range central forces between the ions in simple metals, and between ion cores in non simple metals. Since the outermost core electrons in the simple metals are rigidly bound to the nucleus, their displacement is the same as that of the nucleus, and equation (7) reduces to equation (2).

\section{Application}

Least square fits of the experimental frequencies for the fcc metals, copper and nickel, were initially made by setting $P$ equal to 1 and varying $K$ and $S$. The resulting fits are sufficiently good to warrant their publication. The experimental frequencies for copper were obtained from neutron scattering experiments of Svensson et al (1967) and the adiabatic elastic constants and lattice constant were those used in Svensson's paper. The experimental frequencies, elastic constants and lattice constant for nickel were taken from Birgeneau's (1969) and de Klerk's (1959) papers, respectively. The elastic and lattice constants are shown in table 1 along with the force constants. Figure 1 shows the results for copper, and figure 2 the results for nickel. Two values of the screening parameter $\hat{\lambda}$ were considered; the Bohm-Pines and the Thomas-Fermi. The broken line in 
Table 1. Material constants and model parameters

\begin{tabular}{|c|c|c|c|c|c|c|c|c|c|c|}
\hline \multirow[t]{2}{*}{ Metal } & \multicolumn{3}{|c|}{$\begin{array}{l}\text { Elastic constant } \\
\left(\times 10^{11}{\left.\mathrm{dyn} \mathrm{cm}^{-2}\right)}^{-}\right.\end{array}$} & \multirow{2}{*}{$\begin{array}{l}\text { Lattice } \\
\text { constant } \\
(\AA)\end{array}$} & \multicolumn{4}{|c|}{ Force constants $\left(\times 10^{3} \mathrm{dyn}^{-1}\right)$} & \multirow[t]{2}{*}{$\begin{array}{l}A\left(2 a^{3} \lambda\right)^{-1} \\
\left(\times 10^{11} \mathrm{dyn} \mathrm{cm}^{-2}\right)\end{array}$} & \multirow[t]{2}{*}{ i. } \\
\hline & $C_{11}$ & $C_{12}$ & $C_{44}$ & & $x_{1}$ & $x_{2}$ & $K$ & $S$ & & \\
\hline $\mathrm{Cu}$ & $16 \cdot 85$ & $12 \cdot 15$ & $7 \cdot 55$ & 3.6147 & $-6 \cdot 26$ & $2 \cdot 57$ & $-2980 \cdot 0$ & $-21 \cdot 0$ & $4 \cdot 6$ & BP \\
\hline $\mathrm{Cu}$ & - & - & - & - & 57.74 & $2 \cdot 57$ & $-8250 \cdot 0$ & $-85 \cdot 0$ & 46 & $\mathrm{TF}$ \\
\hline $\mathrm{Ni}$ & $24 \cdot 6$ & $15 \cdot 0$ & $12 \cdot 2$ & $3 \cdot 524$ & $65 \cdot 03$ & $2 \cdot 29$ & $-7772 \cdot 0$ & $-110 \cdot 0$ & $2 \cdot 8$ & TF \\
\hline
\end{tabular}

figure 1 gives the results using the Bohm-Pines value. In an attempt to improve the fit for the (111) direction, the Thomas-Fermi value was used. From figure 1, it is seen that the fit for $(100)_{\mathrm{L}}$ and $(110)_{\mathrm{L}}$ is improved at the expense of the fit for the $(100)_{\mathrm{T}}$ and (111) . The best overall fit for copper was obtained by using the Bohm-Pines value. The average deviation from the experimental mean values was $2 \%$, while the largest deviation was $3 \%$. For nickel, only the Thomas-Fermi screening parameter was used because preliminary calculations indicated that the frequencies for the (100) and (110) directions could be fitted as well using either value, while the fit to the (111) direction would be somewhat improved using the Thomas-Fermi value. The average deviation for nickel was $2.5 \%$, while the largest deviation was $4 \%$. As can be seen from the figures, the fits are rather similar for the two metals and the overall fit for both metals is good for all three directions with the exception of the discrepancies for the (111) direction at the boundary. It will be interesting to see if this discrepancy is removed by using a value of $P$ less than one. Such an investigation is now underway and the results will be published in the near future.

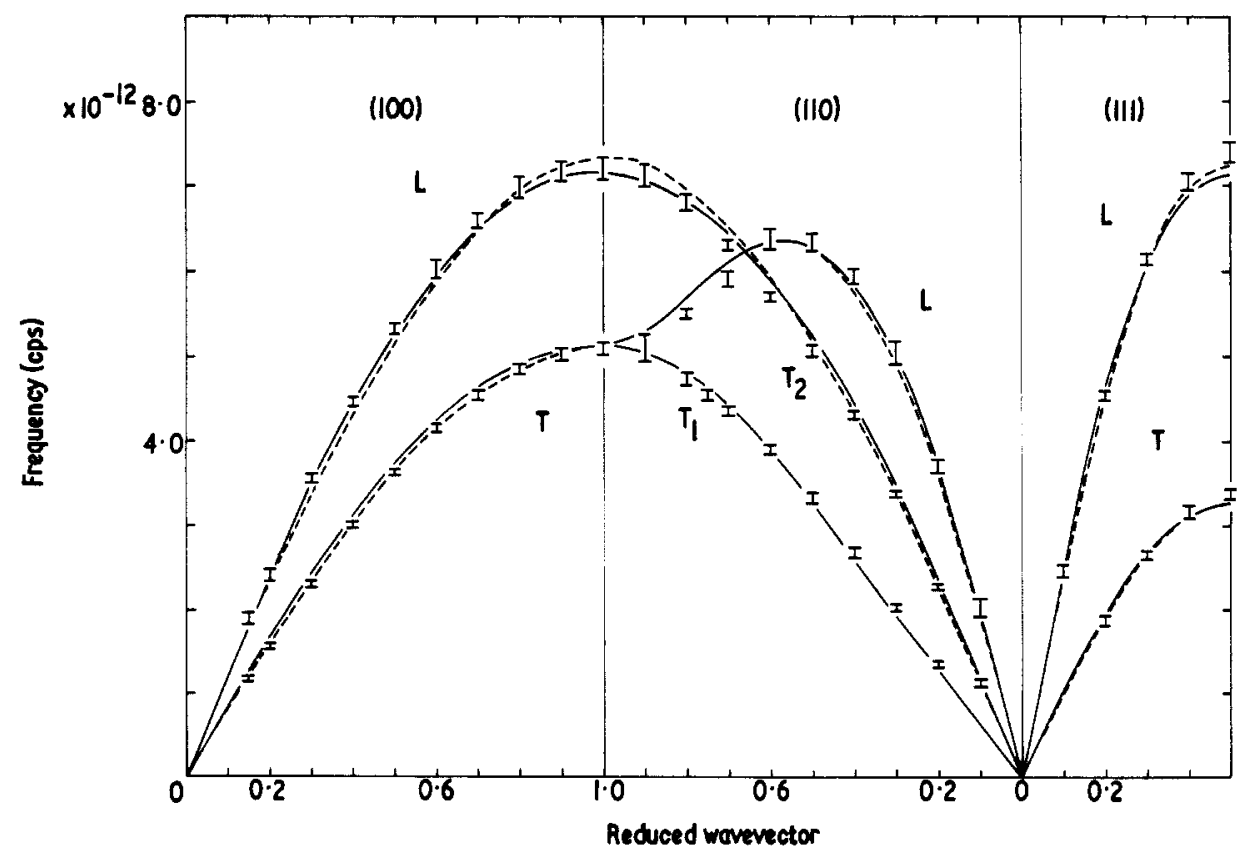

Figure 1. Phonon dispersion curves along symmetric directions for copper. The broken curve is with BP screening parameter and the solid curve is with the TF screening parameter. 


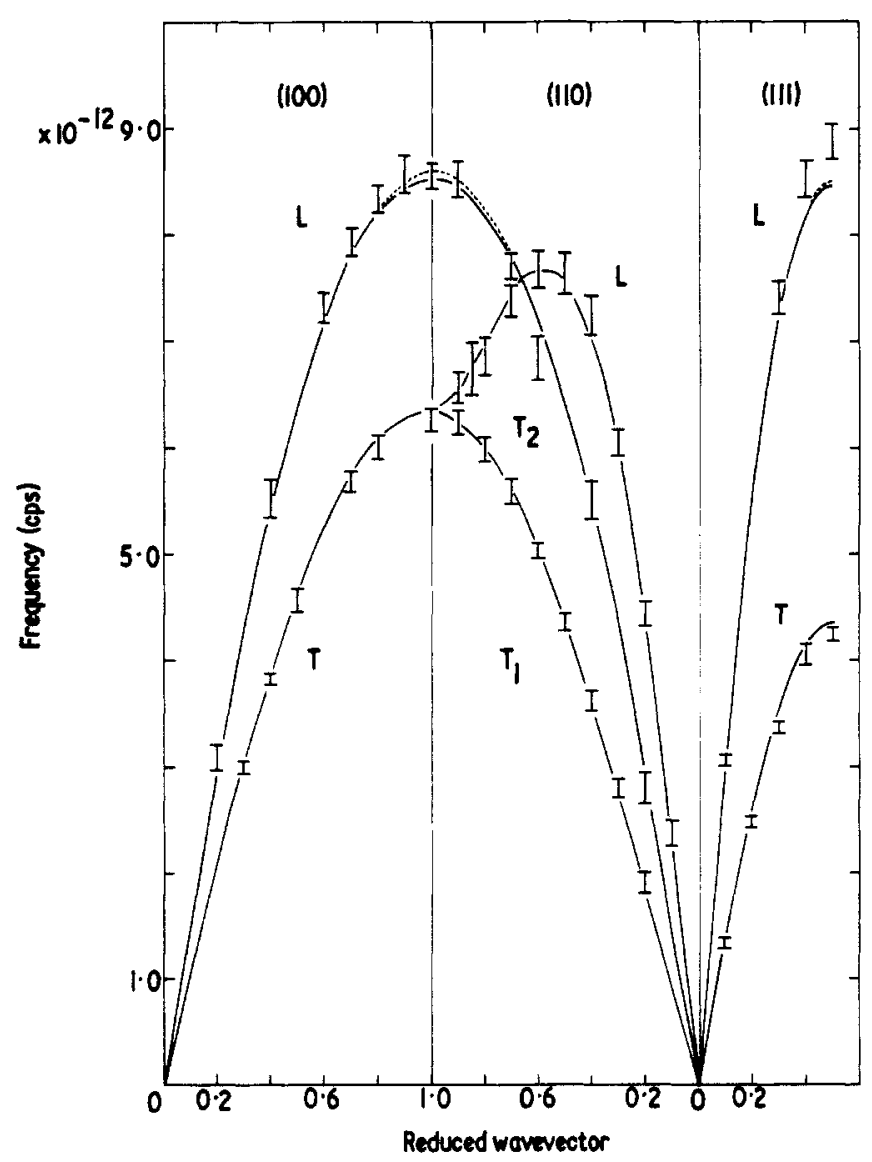

Figure 2. Phonon dispersion curves along symmetric directions for nickel. The solid curve is with TF screening parameter.

\section{Conclusions}

In this paper a very simple model has been proposed for the calculation of the phonon dispersion curves of the noble and transition metals. Preliminary results show that the model may give a very good reproduction of the dispersion curves for the noble and transition metals. It should be remarked that, besides reproducing the phonon dispersion curves, the model may be useful in investigations into the effect of different dielectric functions on the phonon dispersion curves of the noble or transition metals. Furthermore, it may be possible to study the effects of the electronic band structure on the dispersion curves through the dielectric function by considering appropriate dielectric functions modified by the band structure of the metal.

\section{References}

Birgeneau R J 1969 Phys. Ret. 136 A1359-65

de Klerk J 1959 Proc. Phys. Soc. $73337-44$

de Launay J 1956 Solid State Physics ed F Seitz and D Turnbull (New York: Academic Press) vol 2 pp 219 306 